\title{
Chloride extrusion alleviates neuropathic pain
}

The potassium-chloride co-transporter KCC2 is expressed in neurons of the central nervous system (CNS), where it is responsible for maintaining low $\mathrm{Cl}^{-}$concentrations. Loss of activity of KCC2 is a key mechanism underlying several neurological and psychiatric disorders, including epilepsy, schizophrenia and chronic pain. Now, reporting in Nature Medicine, De Koninck and colleagues present KCC2 activators that show promising oral efficacy in a rat model of neuropathic pain.

The maintenance of low $\mathrm{Cl}^{-}$ concentrations is essential for postsynaptic inhibition, and $\mathrm{Cl}^{-}$extrusion is controlled by a family of cation chloride cotransporters including KCC2. KCC2 is particularly attractive as a drug target as its expression is strictly limited to the CNS.

To identify compounds that increase $\mathrm{Cl}^{-}$efflux via $\mathrm{KCC} 2$, the authors developed a fluorometric assay using a $\mathrm{Cl}^{-}$-sensitive indicator in a neuronal cell line (NG-108) that has low KCC2 activity and mimics the pathophysiological situation in neuropathic pain. A high-throughput screen of 92,500 drug-like compounds, followed by a lead optimization campaign, led to the development of CLP257, a small molecule that achieved a maximal $\mathrm{Cl}^{-}$reduction of $40 \%$ in NG-108 cells.

Further in vitro experiments showed that CLP257 selectively modulates KCC2 over other KCC family members, increasing its transport activity by over $60 \%$. CLP257 was then tested in spinal slices from rats with peripheral nerve injury (PNI) - a model of neuropathic pain - as well as spinal slices that were pre-treated with brainderived neurotrophic factor (BNDF), which is known to downregulate KCC2 activity and is used in models of pain hypersensitivity. These experiments demonstrated that CLP257 effectively enhances $\mathrm{Cl}^{-}$transport in mature primary CNS neurons that have reduced KCC2 function. Further biochemical investigations established that CLP257 does not affect overall KCC2 levels, but boosts KCC2 activity by increasing its expression on the cell surface.

Ensuing in vivo experiments showed that local spinal administration of CLP257 to PNI rats normalized electrophysiological responses to mechanical stimuli, and PNI rats that received CLP257 via intraperitoneal injection had normalized paw withdrawal responses to allodynic stimuli. However, the drug displayed poor pharmacokinetics, so the authors designed a carbamate prodrug (CLP290) with a significantly improved plasma half-life. In PNI rats, CLP290 showed a maximal oral efficacy that was comparable to pregabalin, an anticonvulsant drug that is approved for the treatment of neuropathic pain. However, unlike pregabalin, which causes sedation and dizziness and thereby affects motor function, CLP290 had no side

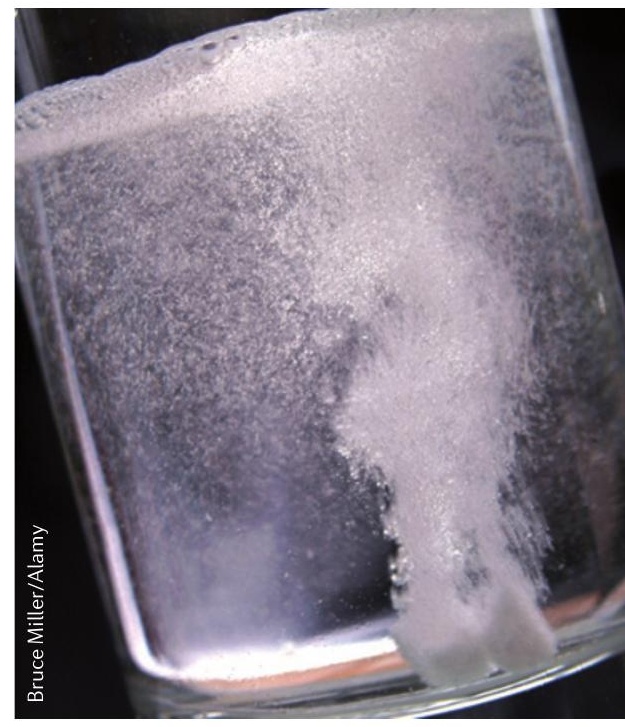

effects. Further toxicity studies after repeated dosing showed excellent tolerability.

These results confirm KCC2 as a promising druggable target and identify $\mathrm{Cl}^{-}$modulation as a mechanism of action for potential new therapeutics. As KCC2 has been implicated in a variety of disorders, compounds targeting this co-transporter might be useful for treating a broad range of neurological and psychiatric conditions.

\section{Alexandra Flemming}

ORIGINAL RESEARCH PAPER Gagnon, M. et al. Chloride extrusion enhancers as novel

therapeutics for neurological diseases. Nature Med. http://dx.doi.org/10.1038/nm.3356 (2013) 